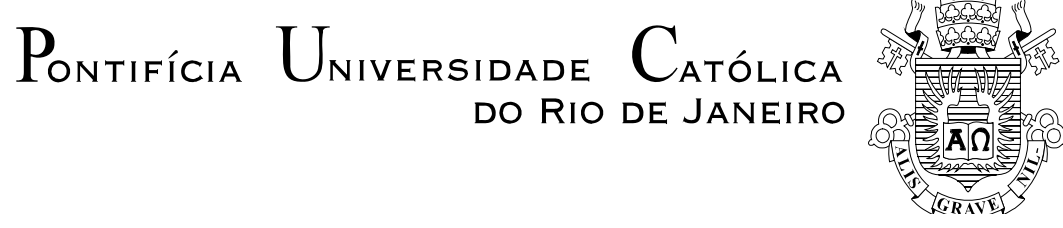

Mariana Rodrigues Coutinho

Gerenciamento Integrado de Riscos de Projetos

Dissertação apresentada como requisito parcial para obtenção do grau de Mestre pelo Programa de Pósgraduação em Engenharia Elétrica do Departamento de Engenharia Elétrica da PUC-Rio.

Orientador: Prof. Álvaro de Lima Veiga Filho

Rio de Janeiro

Setembro de 2010 


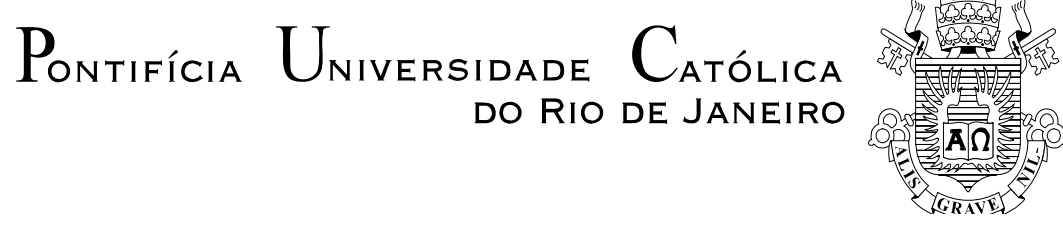

Mariana Rodrigues Coutinho

\title{
Gerenciamento Integrado de Riscos de Projetos
}

Dissertação apresentada como requisito parcial para obtenção do grau de Mestre pelo Programa de PósGraduação em Engenharia Elétrica do Departamento de Engenharia Elétrica do Centro Técnico Científico da PUCRio. Aprovada pela Comissão Examinadora abaixo assinada.

\author{
Prof. Álvaro de Lima Veiga Filho \\ Orientador \\ Departamento de Engenharia Elétrica - PUC-Rio
}

Prof. Leonardo Lima Gomes

Departamento de Administração-PUC-Rio

Prof. Alexandre Street de Aguiar

Departamento de Engenharia Elétrica - PUC-Rio

Prof. José Eugenio Leal Coordenador Setorial do Centro

Técnico Científico 
Todos os direitos reservados. É proibida a reprodução total ou parcial do trabalho sem autorização da universidade, da autora e do orientador.

\section{Mariana Rodrigues Coutinho}

Graduou-se em 2003 em Engenharia de Produção Elétrica pela PUC-Rio.

Ficha Catalográfica

Coutinho, Mariana Rodrigues

Gerenciamento integrado de riscos de projetos / Mariana Rodrigues Coutinho ; orientador: Álvaro de Lima Veiga Filho. - 2010.

$110 \mathrm{f} . ; 30 \mathrm{~cm}$

Dissertação (mestrado) - Pontifícia Universidade Católica do Rio de Janeiro, Departamento de Engenharia Elétrica, 2010.

Inclui bibliografia

1. Engenharia elétrica - Teses. 2. Gerenciamento de riscos de projetos. 3. Simulação de Monte Carlo. I. Veiga Filho, Álvaro de Lima. II. Pontifícia Universidade Católica do Rio de Janeiro. Departamento de Engenharia Elétrica. III. Título. 


\section{Agradecimentos}

Ao orientador Álvaro Veiga Filho, pela motivação, ensinamentos e apoio durante a execução deste trabalho.

Aos membros da minha banca Alexandre Street e Leonardo Lima, pelas contribuições a este trabalho.

Aos meus pais Frederico Coutinho e Maria Angelica Barreto, por me proporcionarem a oportunidade de me capacitar e me desenvolver como pessoa e profissional. São os principais responsáveis por tudo que sou hoje. A minha irma Maria Coutinho pela eterna amizade.

Ao meu marido Manuel Junior, que sempre esteve ao meu lado incondicionalmente e me motivou ao longo do desenvolvimento deste trabalho com muita paciência.

A todos os amigos da Promon. Um especial agradecimento ao Octavio Pieranti, meu grande mentor, pela orientação e motivação para desenvolvimento deste trabalho. Aos orientadores Patricia Sibinelli, Paula Giudicelli e Eric Rosenthal pela constante preocupação em estar sempre abrindo portas e me incentivando academicamente.

As minhas grandes amigas Cecilia Bhering, Carolina Wigg, Cristiane Almeida, Joanna Areal, Sabrina Lobo, Mariana Albuquerque e Mariana Yoshioka pela amizade durante os momentos bons e difíceis.

A PUC-Rio pela bolsa de isenção concedida ao longo da realização deste curso. 


\section{Resumo}

Coutinho, Mariana Rodrigues, Veiga Filho, Álvaro de Lima (Orientador). Gerenciamento Integrado de Riscos de Projetos. Rio de Janeiro, 2010, 110p. Dissertação de Mestrado - Departamento de Engenharia Elétrica, Pontifícia Universidade Católica do Rio de Janeiro.

A dissertação, com o título "Gerenciamento Integrado de Riscos de Projetos", propõe uma metodologia de análise quantitativa de riscos de projetos integrando os riscos financeiros, como variações cambiais, inflação, preços de commodities, à análise quantitativa dos riscos operacionais. No estudo, os riscos operacionais são quantificados através da geração de cenários de fluxo de caixa do projeto utilizando simulação Monte Carlo. Os preços e taxas são simulados através de um modelo econométrico e incorporados aos cenários de fluxo de caixa do projeto, de forma a apresentarmos uma curva de distribuição de probabilidade acumulada da margem do projeto. A metodologia proposta foi aplicada ao projeto de uma termelétrica à carvão. Ao incorporarmos esta metodologia a todos os projetos da empresa, podemos também avaliar os riscos da carteira de projetos, avaliando a liquidez e saúde financeira da empresa.

\section{Palavras-chave}

Gerenciamento de riscos de projetos; simulação de Monte Carlo. 


\section{Abstract}

Coutinho, Mariana Rodrigues, Veiga Filho, Álvaro de Lima (Advisor). Project Risk Management. Rio de Janeiro, 2010, 110p. MSc Dissertation - Departamento de Engenharia Elétrica, Pontifícia Universidade Católica do Rio de Janeiro.

The dissertation, entitled "Integrated Risk Management Project" proposes a methodology for quantitative analysis of project risks by integrating the financial risks such as exchange rate fluctuations, inflation, commodity prices, the quantitative analysis of operational risks. In the study, operational risks are measured through the generation of cash flow scenarios for the project using Monte Carlo simulation. Prices and fees are simulated using an econometric model and incorporated into the scenarios of project cash flow in order to introduce a distribution curve of cumulative probability of the margin of the project. The proposed methodology was applied to the design of a thermal coal. By incorporating this methodology to all projects within the company, we can also assess the risks of the project portfolio, assessing liquidity and financial health of the company.

\section{Keywords}

Risk management; Monte Carlo simulation. 


\section{Sumário}

1 Introdução 11

1.1 O Conceito de Risco 13

$\begin{array}{ll}1.2 \text { Metodologia de Medição de Risco } & 15\end{array}$

2 Metodologia de Medição de Riscos para Projetos 18

2.1 Identificação das Variáveis de Interesse, Fatores de Riscos e Premissas

2.2 Modelo de Cálculo para as Variáveis de Interesse: Fatores de Riscos Operacionais e Financeiros

2.3 Formulação de um Modelo Probabilístico para os Fatores de Riscos

2.4 Geração de Cenários para os Fatores de Riscos

2.5 Definição das Distribuições de Probabilidades das Variáveis de Interesse e suas Medidas de Riscos

3 Metodologia de Gerenciamento de Riscos

3.1 Processo de Gerenciamento de Riscos - Abordagem Clássica 24

3.2 Planejar o Gerenciamento de Riscos 25

$\begin{array}{ll}3.3 \text { Abrangência do Processo } & 27\end{array}$

3.4 Etapas do Gerenciamento de Riscos - Principais Técnicas e Ferramentas

4 Metodologia de Gerenciamento Integrado de Riscos 37

4.1 Identificação de Riscos $\quad 27$

4.2 Análise Qualitativa $\quad 39$

4.3 Análise Quantitativa 41

4.4 Planejamento de Respostas aos Riscos 48

4.5 Monitoramento e Controle 49

5 Estudo de Caso - UTE Padrão 51

5.1 Descrição do Projeto $\quad 51$

5.2 Condições do Contrato 56

5.3 Aplicação da Metodologia de Gerenciamento Integrado de Riscos UTE Padrão

6 Conclusão $\quad 83$

7 Referências bibliográficas $\quad 86$

8 Anexos $\quad 88$ 


\section{Lista de figuras}

$\begin{array}{ll}\text { Figura 1 - Distribuição de probabilidade de uma variável X } & 14\end{array}$

$\begin{array}{ll}\text { Figura 2 - Histograma ou distribuição empírica } & 14\end{array}$

$\begin{array}{ll}\text { Figura } 3 \text { - Modelo probabilístico } & 16\end{array}$

Figura 4 - Modelo probabilístico risco corporativo 17

$\begin{array}{ll}\text { Figura 5 - Modelo probabilístico de risco do projeto } & 18\end{array}$

Figura 6 - Modelo probabilístico do risco da carteira de projetos 19

Figura 7 - Exemplo de Grafo 20

Figura 8 - Processo de Gerenciamento de Riscos de Projetos 25

$\begin{array}{ll}\text { Figura 9 - Abrangência do processo } & 28\end{array}$

Figura 10 - Exemplo de fluxograma $\quad 31$

Figura 11 - Exemplo de matriz de probabilidade x impacto $\quad 40$

Figura 12 - Exemplo de análise qualitativa 40

Figura 13 - Exemplo de matriz de relação EAP x RBS 41

Figura 14 - Processo de analise quantitativa $\quad 42$

Figura 15 - Exemplo de distribuição triangular $\quad 42$

Figura 16 - Exemplo de modelo de geração de cenários 43

Figura 17 - Exemplo curva de probabilidade acumulada 44

Figura 18 - Exemplo gráfico de sensibilidade do tipo tornado 44

Figura 19 - Exemplo de gráfico de índice de criticidade 45

Figura 20 - Exemplo de curva de avanço físico probabilístico 45

Figura 21 - Exemplo curva de probabilidade acumulada de margem do projeto 46

Figura 22 - Ilustração de impacto dos índices financeiros no fluxo de caixa 47

Figura 23 - Exemplo de curva de avanço físico probabilístico e $\begin{array}{ll}\text { orçamento probabilístico } & 48\end{array}$

Figura 24 - Exemplo do planejamento de respostas 49

Figura 25 - Exemplo de planilha de monitoramento e controle $\quad 50$

$\begin{array}{ll}\text { Figura } 26 \text { - RBS da UTE Padrão } & 58\end{array}$

Figura 27 - Matriz de probabilidade x impacto $\quad 62$

Figura 28 - Histórico de dados do índice ICC-RJ - Mão de Obra
(Coluna 10)

Figura 29 - Histórico de dados do índice INCC (Coluna 74) 66

$\begin{array}{ll}\text { Figura } 30 \text { - Histórico de dados do índice IPA-OG-DI - Máquinas e } & \\ \text { Equip. (Coluna 32) } & 67\end{array}$

Figura 31 - Histórico de dados do índice ABDIB - RJ 67

Figura 32 - Histórico de dados do índice INPC-IBGE 68

Figura 33 - Histórico de dados do Dólar $\quad 68$

Figura 34 - Cenarios ICC RJ (Col.10) 69

Figura 35 - Cenarios INCC (Col.74) 69

$\begin{array}{ll}\text { Figura } 36 \text { - Cenarios IPA-OG-DI } & 70\end{array}$

$\begin{array}{ll}\text { Figura } 37 \text { - Cenarios ABDIB RJ } & 70\end{array}$

Figura 38 - Cenarios INPC-IBGE $\quad 71$

Figura 39 - Cenarios de dólar $\quad 71$

Figura 40 - Gráfico de sensibilidade do tipo tornado UTE Padrão 72

Figura 41 - Gráfico tornado de índice de criticidade UTE Padrão 73 
Figura 42 - Curva de probabilidade acumulada de prazo do projeto 73

Figura 43 - Curva de probabilidade acumulada de data final do projeto $\quad 74$

Figura 44 - Curva de avanço física probabilística UTE Padrão 75

Figura 45 - Curva de probabilidade acumulada de margem PertMaster $\quad 76$

Figura 46 - Curva de probabilidade acumulada de margem UTE Padrão $\quad 77$

Figura 47 - Histograma de margem UTE Padrão $\quad 77$

Figura 48 - Cenários de saldo de caixa UTE Padrã $\quad 78$

Figura 49 - Ilustração curva de avanço probabilística $\mathrm{x}$ curva de probabilidade acumulada

Figura 50 - Orçamento probabilístico UTE Padrão 


\section{Lista de tabelas}

Tabela 1 - Exemplo tabela de estimativa de probabilidade 39

Tabela 2 - Exemplo de tabela de estimativa de impactos 40

Tabela 3 - Dados técnicos dos principais equipamentos da UTE Padrão 52

Tabela 4 - Riscos identificados UTE Padrão $\quad 59$

Tabela 5 - Tabela de probabilidades $\quad 61$

Tabela 6 - Grau de impactos em custo e prazo 61

Tabela 7 - Resumo riscos identificados $\quad 62$ 